\title{
Does Chemically Modified Curcumin Control the Progression of Periodontitis? A Systematic Review
}

\author{
Esam Dhaifullah ${ }^{\prime}$ \\ Hassan S Seayed' \\ Diana Mostafa ${ }^{1,2}$ \\ Abdul Majeed M Alharbi' \\ Waleed M Alotaibi' \\ 'Department of Preventive Dental \\ Sciences, Vision Colleges, Riyadh, \\ Kingdom of Saudi Arabia; ${ }^{2}$ Clinical \\ Periodontology Department, Faculty of \\ Dentistry, Alexandria University, \\ Alexandria, Egypt
}

Correspondence: Diana Mostafa Clinical Periodontology Department, Faculty of Dentistry, Alexandria University, Alexandria, Egypt

Email dr.dianamostafa@gmail.com
Background: Recently, pharmacologic approaches have been seen in utilizing matrix metalloproteinase inhibitors (MMP-I) to prohibit the destruction of connective tissue accompanied by erythrogenic inflammatory diseases such as periodontitis. However, curcumin characteristics have been described to be effective in reducing inflammatory mediators and matrix metalloproteinase (MMP). But, due to its poor solubility and bioavailability, a chemically modified curcumin (CMC 2.24) has been used.

Objective: The purpose of this research is to review and analyze the animal attempts which investigate the impact of $\mathrm{CMC} 2.24$ on periodontitis.

Materials and Methods: Our study was based on reviewing the English preclinical studies using CMC2.24 on an induced periodontal disease which were published up to 2020, only randomized control trials (RCTs) were included. Databases were used from electronic websites including PubMed, ScienceDirect, and Google scholar.

Results: Seven experimental trials involving 162 rats and 8 dogs were included in the present systematic review. Six studies investigated LPS-induced experimental periodontitis, two of them worked on diabetes-associated periodontitis, while one study worked on naturally occurring periodontitis. All included studies revealed that CMC 2.24 reduced alveolar bone loss as well as inhibited the MMP.

Conclusion: Collectively, we concluded that CMC 2.24 has significant implications in prohibiting the progression of bone loss.

Keywords: chemically modified curcumin, matrix metalloproteinase, cytokines, periodontitis, CMC2.24

\section{Introduction}

Periodontitis (PD) is counted as the highest predominant inflammatory disease encountered in individuals, ${ }^{1}$ where it affects around $50 \%$ of adults over 30 years old around the world. It is distinguished by the immunological breakdown of soft connective tissues and alveolar bone in the existence of microbial pathogenic biofilm inside the periodontal pockets. When it is not managed or ineffectively managed, it conducts progressive tissue-attachment damage, bone resorption and tooth mobility. ${ }^{2,3}$ Worthy to be mentioned that periodontal health transgresses the boundaries of the oral cavity and its correlation with numerous systemic diseases have been documented, particularly endocrine diseases, chronic kidney diseases and cardiovascular diseases. ${ }^{2,3}$ Besides, obesity, stress and smoking were reported to be highly contributed to the progression of PD. ${ }^{4}$

Through the pathogenesis of PD, anaerobic gram-negative bacteria such as P. gingivalis, T. forsythia, P. intermedia and their bacterial products, 
lipopolysaccharide (LPS) or endotoxin trigger the immune reactions, ${ }^{1}$ inducing the periodontal tissue inflammation process. At the lesion of the disease, inflammatory cells including neutrophils and macrophages are enrolled in the infected area, elevating the quantities of cytokines and other pro-inflammatory mediators involving Tumor necrotizing factor (TNF-alpha), Interleukins (IL-1 and IL-6) and the prostaglandins $\left(\mathrm{PGE}_{2}\right)$. Subsequently, excessive collagenolytic enzymes and matrix metalloproteinase (MMP) expression are produced and, the latter action presents in the damage of periodontium and attachment loss including apical migration of junctional epithelium, connective tissues destruction and reduction of bone level. ${ }^{4,5}$

The primary purpose of PD therapeutic strategies is to eliminate the inflammation by diminishing the adherent pathogenic microorganisms but on the other hand, host response plays an important role to complete the natural balance for periodontal health. Scaling and root planning (SRP) is counted as "the gold standard" for the mechanical approach in treating PD via removing the soft and hard microbial deposits. Nevertheless, in some conditions as in deep pockets and unapproachable areas where instrumentation is problematic to handle, auxiliary treatment is required adjunctive to SRP, such as the use of local and systemic antibiotics, antiseptics, and probiotics. ${ }^{6}$ Further data have been exhibited that SRP alone has an imperfect effect on some pathogenic species where total bacterial eradication is not succeeded, as some of these species can inhabit in soft tissues, dentinal tubules, or cementum irregularities, leading to failure of the PD management. ${ }^{7}$

However, some of these pharmacological strategies have a direct effect on the bacterial count or its destructive activity and influence the host response modulation which helps in inhibiting the alveolar bone loss cascade. Therefore recently, it has been seen attempts to use pharmacologic approaches in utilizing matrix metalloproteinase inhibitors (MMP-I) to prohibit the destruction of connective tissue related to inflammatory conditions such as periodontitis. However, natural curcumin characteristics have been reconnoitered to be effective in the remission of inflammatory mediators and MMPs. Curcumin is a natural hydrophobic yellow polyphenol spice that is obtained from the rhizome of Curcuma Longa ${ }^{8-10}$ Many investigations of pre-clinical models and clinical studies ${ }^{11,12}$ concluded that Curcumin is a multipurpose molecule with considerable advantageous impacts on inflammatory infections as it has anti-inflammatory, anti-oxidant and anti-microbial features, ${ }^{8}$ which make it, propounded as a potent adjunctive factor in periodontal therapy. ${ }^{10}$

Because of its inferior bioavailability and short-term life in plasma, it should be delivered in large quantities, which in turn limits its application clinically. ${ }^{13}$ This is why curcumin has been chemically modified to enhance its zinc-binding characteristics, improve its solubility and increase its bioavailability. ${ }^{13}$ However, chemically modified curcumin (CMC 2.24) is a phenylamino carbonyl curcumin which is triketonic in contrast to the diketonic traditional curcumin, exhibiting extraordinary effect in hindering the MMP causing a pathological inhibition of connective tissues to break down and alveolar bone loss. ${ }^{13}$

The objective of our presented study is to review and evaluate the animal studies which investigated the influence of chemically modified curcumin (CMC2.24) experimentally on periodontitis and compare their outcomes regarding analysis of bone loss and inflammatory mediators.

\section{Methods}

\section{Focused Question}

Our systematic assessment was performed following the Preferred Reporting Items for Systematic Review and meta-analysis (PRISMA) guidelines. ${ }^{14}$ The protocol of the present systematic review was registered at PROSPERO with a registration NO of ID $=250936$.

PICOS [Participants, Intervention, Control, Outcomes and Study design] strategy was established (Table 1) to formulate the focused question: "What are the effects of chemically modified curcumin in controlling the progression of periodontitis?" The participants were animals with periodontitis, the intervention was $\mathrm{CMC} 2.24$ administration, control was placebo solution, the outcomes measured the alveolar bone loss, and the study design was in vivo animal studies.

Table I The PICOS Framework

\begin{tabular}{|l|l|}
\hline Participants & $\begin{array}{l}\text { Laboratory Animals with Induced or } \\
\text { Naturally Occurring Periodontitis }\end{array}$ \\
\hline Intervention & CMC2.24 administration \\
\hline Control & No CMC or vehicle only \\
\hline Outcomes & Alveolar bone loss \\
\hline Study design & Vivo animal studies \\
\hline
\end{tabular}

Abbreviation: CMC2.24, Chemically Modified Curcumin. 


\section{Eligibility Criteria}

Only preclinical studies (animal models) using $\mathrm{CMC} 2.24$ on PD were qualified for this systematic review in which randomized control trials (RCTs) were included. While, exclusion criteria included human studies, literature reviews, editorials, letters to the editor, book chapters and studies published in a language other than English.

\section{Search Strategy}

The searches were carried out up to December 2020. The search was conducted in three online databases in 2020: PubMed (http://www.ncbi.nlm.nih.gov), ScienceDirect (https://www.sciencedirect.com), Google Scholar (https:// scholar.google.com). The following search strategy was used: "Periodontitis" AND "Curcumin" AND "Animals" in both PubMed and ScienceDirect. For Google Scholar the following strategy was used: "Periodontitis" "Chemically modified curcumin" "Animal".

\section{Methods of Screening and Selection}

Only vivo studies that investigated the influence of chemically modified curcumin application on periodontitis progression in animals were selected. According to the PD induction protocol, experimental time, species and sample size, there was no limitation.

\section{Search Criteria and Study Selection}

Before starting the study selection process, the reviewers discussed the eligibility criteria. Two authors (ED, HS) individually performed the search and screening process. The selection process is done using pilot-tested forms. The retrieved articles were elected according to abstracts and/or titles and were screened, while the unrelated articles were eliminated according to the eligibility criteria. The reviewers were not blinded to the names of authors and journals. Full texts of researches achieved from the earlier stage were read and assessed individualistically by investigators for involvement. The full texts of the preliminarily eligible studies were analyzed to verify whether the studies fulfilled the eligibility criteria. In addition, we searched the reference lists of the involved studies manually for further articles. All evaluations, including searches, study selection, and data extraction, were performed independently by two reviewers (ED, HS) and checked by a third-party evaluator (DM) in case of disagreement.

\section{Types of Outcome Measure}

The bone loss was assessed by radiographs or Microcomputerized Tomography ( $\mu-\mathrm{CT})$, MMP was measured by blood immunoblotting while cytokines such as IL-1,IL-6 and TNF-alpha were evaluated by Enzymelinked immunosorbent Assay (ELISA).

\section{Analysis and Statistics}

The preliminary objective was to perform a meta-analysis but because of the noticeable inconsistency and heterogeneity of the information of the involved researches, we could not achieve the statistical analysis.

\section{Quality Assessment and Bias Risk}

The assessment criteria of the studies' quality were reformed from the Strengthening the Reporting of Observational Studies in Epidemiology Statement (STROBE). Seven parameters were reflected the most noteworthy ones in the perspective of our article within a checklist. ${ }^{15}$ The adopted STROBE checklist contained; the study design, study participants, the sample size, variables, potential confounders, outcomes and statistical analysis test. The presence or absence of each criterion was documented as either "Yes" or "NO". Henceforth, each research could have the highest total of 7 . Then, we totaled the scores and graded the quality of methodology as low (0-3), acceptable (4-5), and high (6-7).

Additionally, bias risk was evaluated independently by two investigators (ED, HS) by using the SYRCLE (Systematic Review Center for Laboratory Animal Experimentation) approach as shown in Table 2. Both reviewers judged 10 items related to selection bias, performance bias, detection bias, attrition bias, reporting bias and other biases. When there were any disagreements between both reviewers, they consulted a third reviewer (DM) for a final decision. Items to be judged included allocation sequence generation (selection bias), similar baseline characteristics (selection bias), allocation concealment (selection bias), random housing (performance bias), blinding of investigators (performance bias), random outcome assessment (detection bias), blinding of outcome (detection bias), incomplete outcome data (attrition bias), free from selective reporting (reporting bias) and free from other problems.

The entries were considered as yes/no/unclear. A "yes" judgment indicated low bias risk, and a "no" judgment indicated high bias risk. An "unclear" judgment indicated 


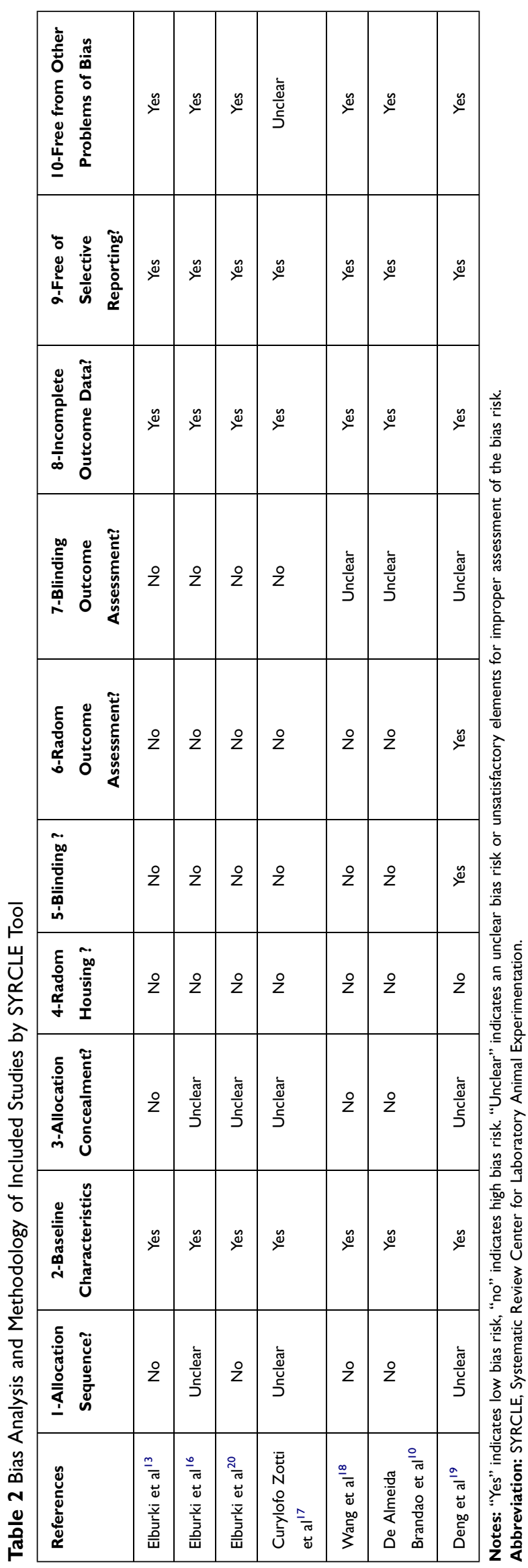

an unclear bias risk or unsatisfactory elements for improper assessment of the bias risk.

\section{Results}

\section{Study Identification and Screening}

Figure 1 (Flow chart of the screening process) presents the PRISMA flowchart for recognition of related included studies. The initial manual and electronic search in PubMed, ScienceDirect and Google Scholar databases retrieved a total of 130 articles. Seventy-five studies remained after eliminating the duplicates. Out of the 75 articles, 23 were excluded for the utilize of curcumin alone, 43 were excluded because of using other substances. After the initial screening phase, 9 studies required full-text reading for assessing eligibility. Two studies were excluded due to unclarity, only 7 articles were included in this study.

\section{General Characteristics}

All authors investigated the general characteristics of the involved studies (Table 3). Seven experimental trials involving 162 rats and 8 dogs were concerned in this systematic review over 14-90 days. ${ }^{10,13,16-20}$ Five articles were published in the USA and two studies were conducted in Brazil. Besides, all studies are contemporary, as the oldest of them was published in 2014.

Six studies of the selected studies investigated LPSinduced experimental periodontitis, ${ }^{10,13,16-20}$ two studies of them ${ }^{14,18}$ worked on diabetes-associated periodontitis, while one study ${ }^{19}$ worked on naturally occurring periodontitis. All rodent animals used in six studies ${ }^{10,13,16-18,20}$ were male Holtzman rats, which were subjected to bilateral palatal injections of the first molars; $30 \mu \mathrm{g}$ of lipopolysaccharide (LPS) two weeks, ${ }^{16-18}$ three weeks, ${ }^{20}$ four weeks. ${ }^{10,13}$ Escherichia coli diluted in phosphate-buffered saline (PBS) was injected palatally $(3 \mu \mathrm{L})$ using am10 $\mu \mathrm{L}$ micro-syringe. The opposite sides were received injected with the same quantity of PBS to be considered as a control group (split-mouth study). Only two studies ${ }^{13,16}$ were subjected to the injection of the intravenous tail with streptozotocin $(70 \mathrm{mg} / \mathrm{kg})$ to provoke diabetes in rats. The effect of CMC $2.24(30 \mathrm{mg} / \mathrm{kg})$ was evaluated by daily oral administration ${ }^{13,16,20}$ while two studies compared the effect of CMC 2.24 and natural curcumin. ${ }^{17,18}$ On the other hand, one study ${ }^{10}$ used various doses of CMC2.24 on rats with 0 (controlled), 1, 3, 10, and $30 \mathrm{mg} / \mathrm{kg}$. 


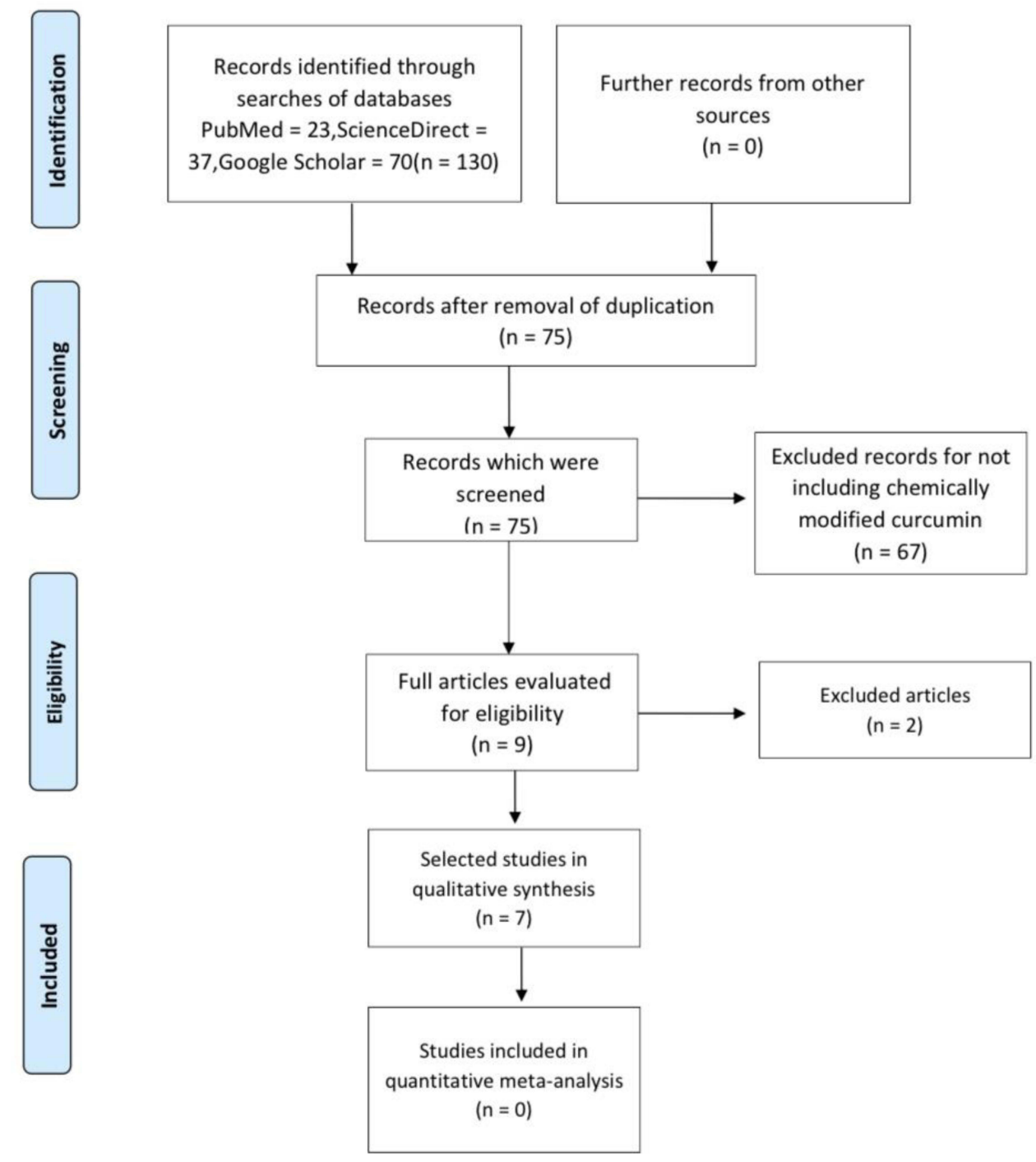

Figure I Article selection flow chart of screening process.

However, only one study ${ }^{19}$ other than the mentioned six studies used large (dog) animals with periodontitis treated orally by $10 \mathrm{mg} / \mathrm{kg}$ of $\mathrm{CMC} 2.24$ (one capsule/ day). Besides, it was the only study which mentioned that scaling and root planning were done before CMC2.24 administration. In addition, it was the only study that assessed the gingival index (GI), plaque index (PI), crevicular gingival fluid flow and CAL before and after CMC2.24 administration.

\section{Main Outcome}

In all recruited studies, bone analysis was performed either by microcomputed tomography where information obtained as a percentage of the fraction of the bone volume ${ }^{10,16,17}$ or by morphometric analysis where datum introduced as a distance in $\mathrm{mm}$ from the cementoenamel junction to the crest of the alveolar bone. ${ }^{13,16-19}$ All included studies revealed that CMC 2.24 administration diminished bone loss significantly. However, one study ${ }^{17}$ showed that natural curcumin did not reduce the destruction of alveolar bone in comparison to CMC 2.24. Two studies ${ }^{13,20}$ found that CMC $2.24 \mathrm{had}$ an unnoticeable influence on the hyperglycemia severity during the protocol of the treatment duration (Table 4). Six studies ${ }^{10,13,16,18-20}$ exhibited that the systemically administered CMC 2.24 reduced the Pro and activated MMP-2 and MMP-9 in gingiva and MMP-9 only in plasma whereas CMC 2.24 had no effect on MMP-2. In addition, the studies $^{10,13,16,18-20}$ displayed that the administration of CMC2.24 is significantly reducing the production of inflammation-associated cytokines IL-1 $\beta,{ }^{13,16,18,20}$ IL- $6,{ }^{13,19,20}$ $\mathrm{TNF}-\alpha,{ }^{10,13,16,19} \mathrm{IL}-10^{10,13,16,19}$ in gingiva and serum to normal levels in both experimental models. 


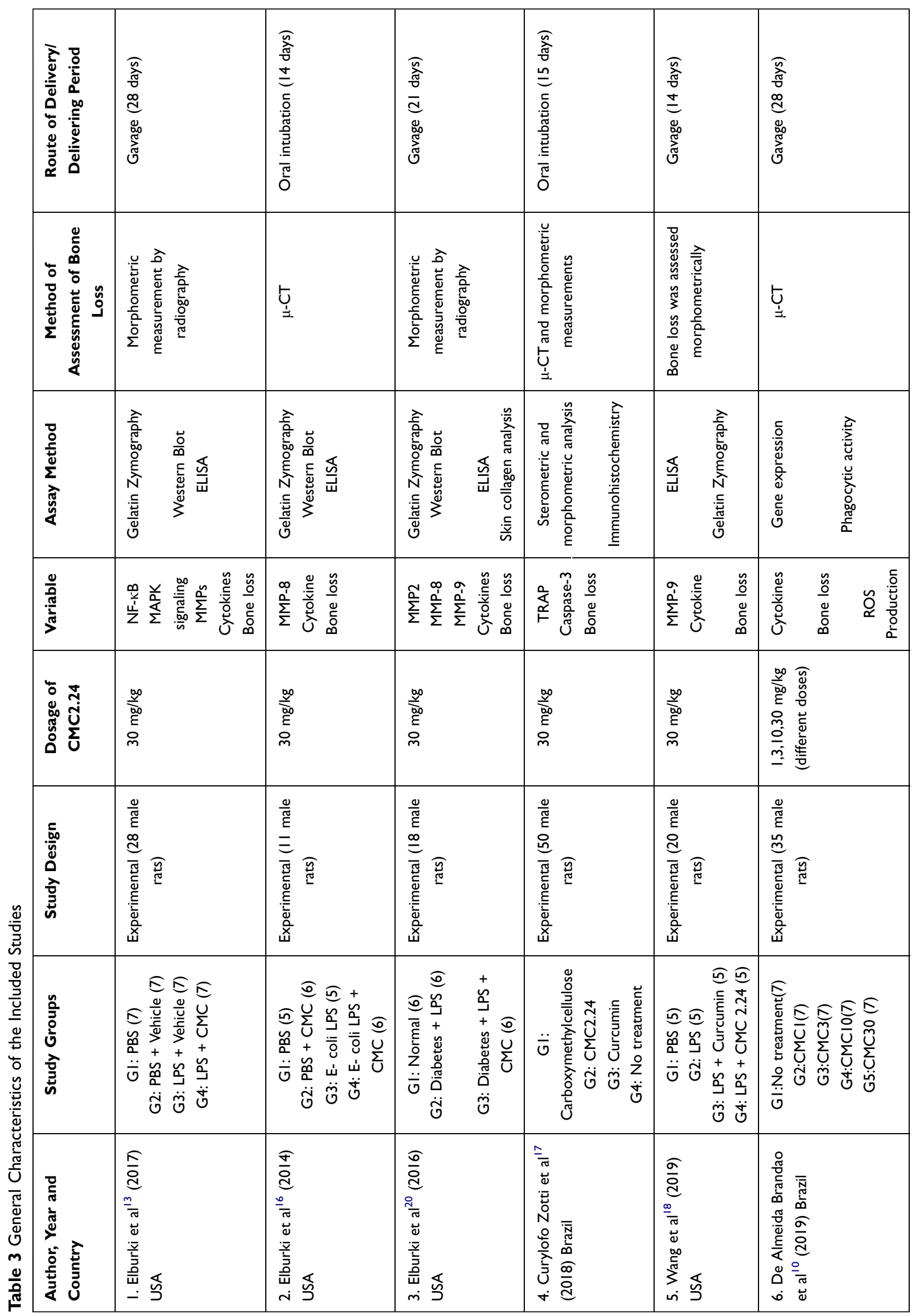




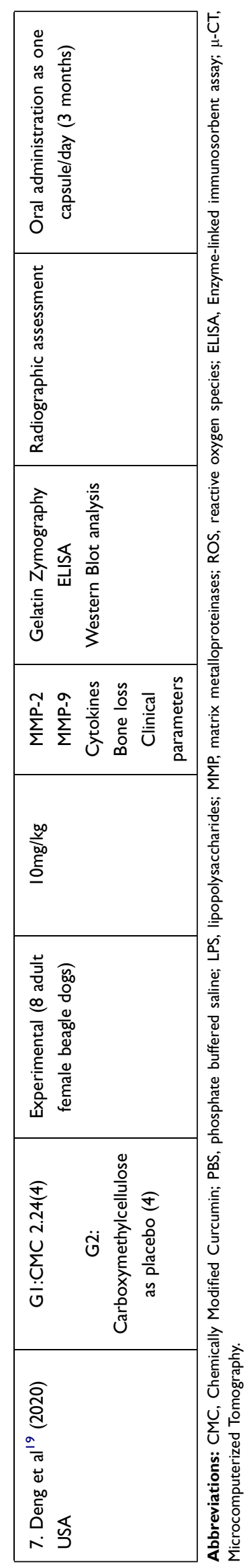

\section{Quality and Risk of Bias}

Our outcomes of the STOBE-based assessment were shown in Table 5. Generally, the quality of the related preclinical studies was high, with a summed score of 6 . Bias analysis of the results was displayed in Table 2, where most of the researches showed a high risk of bias. Four articles ${ }^{10,13,18,20}$ did not report random sequence generation, and this item considered unclear in 3 studies, ${ }^{16,17,19}$ also 3 articles $^{10,13,18}$ did not document the allocation concealment. The authors reported that the animals were randomly allocated to the study groups without declaring the method of sequence generation and allocation concealment. All studies started their experiments with similar characteristics of animals either the weight or age.

All included studies ${ }^{10,13,16-20}$ did not provide any information about the random housing of the animals during the experiments. Besides, it was not possible to blind the investigators and there was no blinding of outcome assessment in all articles except for one article. ${ }^{19}$ All involved studies stated incomplete outcome data and selective reporting. They were considered free of attrition and reporting bias. Lastly, six studies ${ }^{10,13,16-20}$ considered free of other problems of bias except for one study ${ }^{17}$ considered as unclear. Types of bias were presented in Figure 2 in terms of percentages of the involved researches. However, a meta-analysis could not be done as included studies were classified as having a "high risk of bias". Funnel plots could not be prepared due to the low quantity of included articles (less than 10 studies).

\section{Discussion}

Seven preclinical studies were included in this systematic review. This review of the literature revealed a paucity of experimental studies evaluating the effect of $\mathrm{CMC} 2.24$ on periodontitis, there was a consensus among the included seven studies confirming the impact of $\mathrm{CMC} 2.24$ on bone loss reduction and inflammatory markers, inhibiting the activity against MMPs and proinflammatory cytokines. The outcomes of the seven included studies confirmed that administration of chemically modified curcumin has an anti-inflammatory effect which suggests it as an efficacious treatment in reducing bone resorption.

All involved investigations had been conducted on animal models so they cannot be interpreted as human clinical trials. Six studies ${ }^{10,13,16-18,20}$ worked on rats with the same dosage of $30 \mathrm{mg} / \mathrm{kg}$ in the administration while 
Table 4 Main Outcomes and Conclusions of the Included Studies

\begin{tabular}{|c|c|c|}
\hline Study & Main Results & Conclusion \\
\hline $\begin{array}{l}\text { Elburki et al } \\
2017\end{array}$ & $\begin{array}{l}\text { The CMC } 2.24 \text { significantly minimized the release of MMPs, } \\
\text { inflammatory cytokines and bone loss. }\end{array}$ & $\begin{array}{l}\text { The CMC } 2.24 \text { had noticeable anti- inflammatory influence } \\
\text { where it reduced the MMP-9 production and loss of alveolar } \\
\text { bone as well as the activation of NF- } \mathrm{KB}(\mathrm{p} 65) \text { and p } 38 \text { MAPK } \\
\text { in the induced periodontitis models. }\end{array}$ \\
\hline $\begin{array}{l}\text { Elburki et al } \\
2016\end{array}$ & $\begin{array}{l}\text { In LPS-induced periodontitis, measurements were lowered to } \\
\text { normal levels based on either morphometric }(=0.003) \text { or } \mathrm{UCT} \\
(=0.008) \text { analysis. Also, MMPs and cytokines showed } \\
\text { reduction. }\end{array}$ & $\begin{array}{l}\text { The CMC } 2.24 \text { had an effective role on bone loss and } \\
\text { inflammatory mediators in the experimental PD. }\end{array}$ \\
\hline $\begin{array}{l}\text { Elburki et } \mathrm{al}^{20} \\
2016\end{array}$ & $\begin{array}{l}\text { CMC } 2.24 \text { consumption in diabetic animals with induced } \\
\text { periodontitis impaired the resorption of alveolar bone and } \\
\text { diminished the inflammation severity with local and systemic } \\
\text { factors. }\end{array}$ & $\begin{array}{l}\text { The CMC } 2.24 \text { was an effective therapeutic pleiotropic MMP } \\
\text { inhibitor. }\end{array}$ \\
\hline $\begin{array}{l}\text { Curylofo-Zotti } \\
\text { et al }{ }^{17} 2018\end{array}$ & $\begin{array}{l}\text { Both CMC2.24 and curcumin showed a noticeable decrease of } \\
\text { the inflammatory reactions, but } \mu C T \text { analysis displayed that } \\
\text { only CMC2.24 decreased bone loss and the amount of } \\
\text { osteoclasts. }\end{array}$ & $\begin{array}{l}\text { The CMC2.24 is more superior than curcumin as CMC2.24 } \\
\text { had the capability to reduce alveolar bone loss in the PD } \\
\text { animals. }\end{array}$ \\
\hline $\begin{array}{l}\text { Wang et } \text { al }^{18} \\
2019\end{array}$ & $\begin{array}{l}\text { The CMC2.24 decreased the pathological loss of alveolar bone } \\
\text { by } 80-90 \%(P<0.01) \text {, while curcumin enhance }(P<0.05) \text { or } \\
\text { showed no impact on alveolar bone resorption. }\end{array}$ & $\begin{array}{l}\text { The efficacy of MMP-9 inhibitor was related to the ability of } \\
\text { CMC } 2.24 \text { (but not curcumin) to constrain the resorption of } \\
\text { alveolar bone in rats with PD. }\end{array}$ \\
\hline $\begin{array}{l}\text { De Almeida } \\
\text { Brandao et al } \\
2019\end{array}$ & $\begin{array}{l}\text { The CMC2.24 with Img/kg discontinued the bone loss, } \\
\text { osteoclastogenesis, and TNF-alpha in vivo, ie no dose- } \\
\text { dependent influence. In vitro, CMC2.24 decreased levels of } \\
\text { TNF- } \alpha \text { and IL-10, constrained phagocytosis and motivated the } \\
\text { formation of ROS where the dose-dependent impact was } \\
\text { observed only in the production of ROS. }\end{array}$ & $\begin{array}{l}\text { Low doses of oral CMC2.24 were appropriate to constrain } \\
\text { alveolar bone loss. }\end{array}$ \\
\hline $\begin{array}{l}\text { Deng et al }{ }^{19} \\
2020\end{array}$ & $\begin{array}{l}\text { The CMC2.24 decreased GI, GCF flow, PD compared to } \\
\text { placebo. Besides,it decreased MMP-9 and MMP-2 in } \\
\text { periodontal tissues, reduced loss of alveolar bone and } \\
\text { diminished IL-I } \beta \text {. Cell-signaling molecules, TLR-2 (but not } \\
\text { TLR-4) and P38 MARK which in turn reduces inflammation } \\
\text { and collagenolysis. }\end{array}$ & $\begin{array}{l}\text { CMC2.24 reduced clinical features of periodontitis as it } \\
\text { decreased the MMPs, inflammatory cytokines and cell-signaling } \\
\text { molecules. }\end{array}$ \\
\hline
\end{tabular}

Abbreviations: CMC, Chemically Modified Curcumin; PD, periodontitis; LPS, lipopolysaccharide; MMP, matrix metalloproteinases; ROS, reactive oxygen species; TNF, Tumor necrotizing factor; IL, interleukin; $\mu$-CT, micro-computed tomography; NF- $\mathrm{B}$, Nuclear factor kappa B; GI, gingival index; GCF, gingival crevicular fluid.

one study ${ }^{19}$ worked on dogs with the administration of $10 \mathrm{mg} / \mathrm{kg}$ of CMC2.24. Toxicity and weight loss, as well as other harmful effects, were not documented for CMC 2.24 administration in any of the included studies, also the benefits of higher/lower doses are left undemonstrated.

Out of the seven studies, only two studies ${ }^{17,18}$ reviewed the effect of natural curcumin in comparison to CMC 2.24. When Curylofo-Zotti et $\mathrm{al}^{17}$ compared CMC 2.24 and curcumin, they concluded that administration of curcumin and CMC2.24 expressively hindered the local inflammatory actions in the LPS-induced periodontitis models. Interestingly, CMC2.24 decreased inflammatory markers, number of osteoclasts and bone resorption significantly. On the other hand, curcumin alone prohibited the apoptosis of periodontal tissues and osteocytes in the existence of inflammation or its absence.

This is in agreement with Wang et $\mathrm{al}^{18}$ who exhibited that CMC 2.24 consumption was further efficient than curcumin in restraining IL-1 and reduction of MMP-9 secretion by $78 \%$ in contrary to curcumin which was not ineffective. Besides, they found that CMC 2.24 suppressed the pathological loss of the alveolar bone, in contrast to curcumin which increased or had no impact on the loss of alveolar bone. This is maybe because $\mathrm{CMC} 2.24$ is more 
Table 5 STROBE-Based Quality Analysis of the Included Studies

\begin{tabular}{|c|c|c|c|c|c|c|c|c|}
\hline Ref. & $\begin{array}{l}\text { Study } \\
\text { Design }\end{array}$ & Participants & $\begin{array}{l}\text { Sample } \\
\text { Size }\end{array}$ & $\begin{array}{c}\text { Variable } \\
\text { Description }\end{array}$ & $\begin{array}{l}\text { Potential } \\
\text { Confounder }\end{array}$ & Measurements & $\begin{array}{l}\text { Statistical } \\
\text { Analysis }\end{array}$ & $\begin{array}{l}\text { Total } \\
\text { Scores }\end{array}$ \\
\hline Elburki et al ${ }^{13} 2017$ & I & I & 1 & I & 0 & I & I & 6 \\
\hline Elburki et al ${ }^{16} 2014$ & I & I & 1 & I & 0 & I & I & 6 \\
\hline Elburki et $\mathrm{al}^{20} 2016$ & I & I & I & I & 0 & I & I & 6 \\
\hline $\begin{array}{l}\text { Curylofo-Zotti } \\
\text { et } \text { al }^{17} 2018\end{array}$ & I & I & I & I & 0 & I & I & 6 \\
\hline Wang et al ${ }^{18} 2019$ & I & I & 1 & I & 0 & 1 & I & 6 \\
\hline $\begin{array}{l}\text { De Almeida Brandao } \\
\text { et al }{ }^{10} 2019\end{array}$ & I & I & 1 & I & 0 & 1 & I & 6 \\
\hline Deng et a $\left.\right|^{19} 2020$ & I & I & 1 & I & 0 & I & I & 6 \\
\hline
\end{tabular}

Abbreviation: STROBE, Strengthening the Reporting of Observational Studies in Epidemiology.

soluble than curcumin in water-based vehicles which has 3 zinc-binding moieties that can constrain MMP. Also, the high bioavailability of CMC2.24 may be accountable for the weakening of the bone resorption cascades, where there was a reduction in the osteoclast quantity in CMC2.24- administrated models which propose an influence on the differentiation of osteoclasts. ${ }^{18}$

However, Elburki et al ${ }^{16}$ demonstrated the efficacy of CMC 2.24 in an experimental split-mouth study design on 11 male rats, where they found that a substantial diminution of loss of alveolar bone in the LPS-induced periodontitis to the levels observed in the control models and complete suppression of the pathological release of the
MMP-2, MMP-9, and IL-1 $\beta$ levels. Besides, CMC 2.24 decreased the effect of the local inflammatory infection on systemic biomarkers with no adverse influences on the mediators of constitutive connective tissue turnover.

In 2016, Elburki et $a^{13}$ conducted a study on diabeticinduced rats using intravenous injection of streptozotocin and non-diabetic rats (control models) and revealed that systemic consumption of CMC 2.24 of diabetic rats with periodontitis considerably constrained loss of bone, inflammatory process, connective tissue destruction and hyperglycemia, but there were no remarkable effects on the constitutive MMPs necessary for the physiological turnover of the connective tissues.

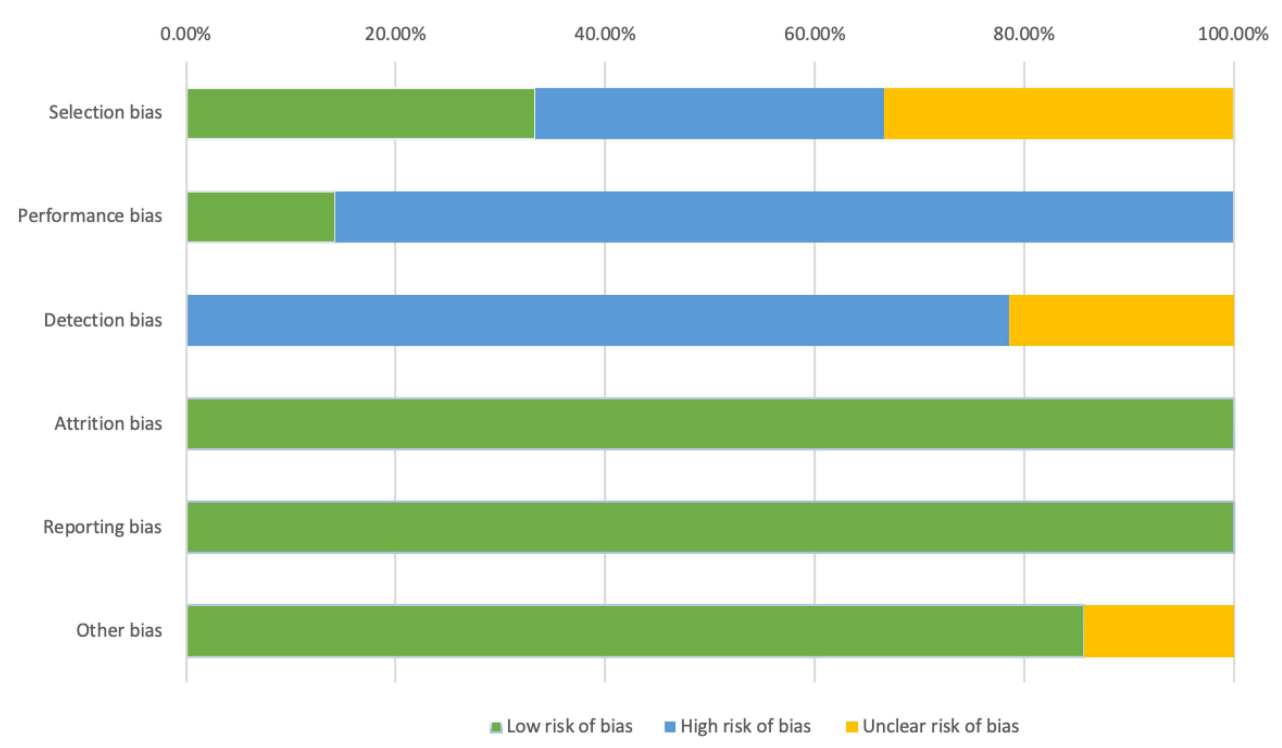

Figure 2 Summary of the bias types of the included studies using the SYRCLE approach. 
While in 2017 Elburki et al, ${ }^{20}$ represented the local and systemic influences separately, which were displayed by bacterial antigen stimulus (LPS) and diabetes-associated immune dysregulation, in 2 independent studied rat models and evaluated the effect of CMC 2.24 on NF- $\mathrm{KB}$ and MAPK signaling and cytokine formation. They discovered that CMC 2.24 had marked anti-inflammatory actions, considerable reduction of loss of alveolar bone, consistently diminished MMP-9 and inhibition of NF- $\mathrm{kB}$ p65 and $\mathrm{p} 38$ MAPK in both models. Moreover, the reduction of pro-inflammatory IL-1 $\beta$ and IL-6 production was observed. Also, they found that CMC 2.24 suppressed levels of TNF- $\alpha$ and IL-10 in the severe form of LPSinduced models of periodontitis, which in turn make CMC2.24 proposed as a potential therapy in the management of periodontal diseases.

However, De Almeida Brandao et al ${ }^{10}$ compared various doses of $\mathrm{CMC} 2.24$ on rats for 28 days and they revealed that oral administration of a low dose of CMC2.24 (1 mg/kg/day) was appropriate to discontinue the resorption of the bone, osteoclastogenesis, and inflammation accompanied with PD. Also, they reported that there was no dose-dependent effect on bone loss except for reactive oxygen species (ROS) production.

Only one preclinical study by Deng et $\mathrm{al}^{19}$ investigated CMC2.24 on large animal models, where CMC2.24 was given orally as one capsule/day to 8 dogs with naturally occurring periodontitis after mechanical debridement. The authors chose dog models to increase the similarity of the periodontitis features to humans, they concluded that CMC2.24 remarkably reduced clinical parameters of $\mathrm{PD}$, as well as diminished the loss of alveolar bone, in comparison to placebo utilization, even in the nonexistence of pathogenic biofilm. They reported that $\mathrm{CMC} 2.24$ suppressed the hostmediated-destructive activity by decreasing the inflammatory mediator levels, prohibiting the pathological alteration of inactive to activate MMP-9, suppressing the excessive levels of MMP-2 and decreasing inflammatory signals by reduction of TLR-2 and P38 MARP expression.

Six studies ${ }^{10,13,16-18,20}$ were have assented that CMC2.24 had the ability to decrease the destructive and inflammatory cascades of periodontitis, but there was a high risk of bias because of the indefinite information in the reviewed articles and inability to make blinding of contributors, personals and assessment of outcomes. Although six studies ${ }^{10,13,16-18,20}$ involved the same type of animals (rats) with similar ages, some unrecognized variances could be present such as subclinical infections which may influence their inflammatory response. ${ }^{21}$ Besides, the difference in the used protocols, evaluation methods, evaluation periods and drug concentrations cause divergences that increase the results' bias and the external validity.

Nevertheless, further evidence and more animal investigations are advised to try different doses to check whether if higher doses are either harmful or beneficial, different types of experimental animals should be considered in future studies. Routes of administration of CMC2.24 should also be varied to check for different results, such as Intraperitoneal and subcutaneous routes.

\section{Conclusion}

Collectively, we concluded from the available documentation of the preclinical studies that CMC 2.24 has a conspicuous biological influence on the enhancement of the periodontal health and the inhibition of PD progression by diminishing the pro-inflammatory mediators levels, modulating the MMP-I mechanism and reducing the alveolar bone loss. The outcomes of this systematic review suggested CMC 2.24 as an effective pharmacological modality adjunctive to SRP in the management of periodontal diseases. However, additional preclinical animal studies with longer duration of follow-up visits are highly suggested to be addressed and comparing different approaches is advocated before shifting to human research.

\section{Disclosure}

The authors report no conflicts of interest in this work.

\section{References}

1. Van Dyke TE, Van Winkel Hoff AJ. Infection and inflammatory mechanisms. $J$ Periodontol. 2013;84:S1-S7. doi:10.1902/ jop.2013.1340018

2. Casanova L, Hughes F, Preshaw P. Diabetes and periodontal disease. BDJ Team. 2015;1:15007. doi:10.1038/bdjteam.2015.7

3. Isola G, Polizzi A, Alibrandi A, Williams RC, Lo Giudice A. Analysis of galectin-3 levels as a source of coronary heart disease risk during periodontitis. J Periodontal Res. 2021;56:597-605. doi:10.1111/ jre. 12860

4. Andrade EF, Orlando DR, Araújo AMS, et al. Can resveratrol treatment control the progression of induced periodontal disease? A systematic review and meta-analysis of preclinical studies. Nutrients. 2019;11:953. doi:10.3390/nu11050953

5. Gu Y, Ryan ME. Overview of periodontal diseases: causes, pathogenesis, and characteristics in Periodontal Diseases and Overall Health: a clinician's guide. In: Williams R, Genco R, editors. Professional Audience Communications. PA, USA: Yardley; 2009:5-23.

6. Ikram S, Hassan N, Raffat MA, Mirza S, Akram Z. Systematic review and meta analysis of double-blind, placebo-controlled, randomized clinical trials using probiotics in chronic periodontitis. JICD. 2018;9: e12338. doi:10.1111/jicd.12338 
7. Colombo AP, Teles RP, Torres MC, et al. Effects of non-surgical mechanical therapy on the subgingival microbiota of Brazilians with untreated chronic periodontitis: 9-month results. J Periodontol. 2005;76:778-784. doi:10.1902/jop.2005.76.5.778

8. Suhag A, Dixit J, Dhan P, Prakash D. Role of curcumin as a subgingival irrigant: a pilot study. PERIO. 2007;4(2):115-121.

9. Shehzad A, Park JW, Lee J, Lee YS. Curcumin induces radio-sensitivity of in vitro and in vivo cancer models by modulating pre-mRNA processing factor 4 (Prp4). Chem Biol Interact. 2013;206:394-402. doi:10.1016/j.cbi.2013.10.007

10. De Almeida Brandao D, Spolidorio LC, Johnson F, et al. Doseresponse assessment of chemically modified curcumin in experimental periodontitis. J Periodontol. 2019;90:535-545. doi:10.1002/ JPER.18-0392

11. Sharma RA, Gescher AJ, Steward WP. Curcumin: the story so far. Eur J Cancer. 2005;41:1955-1968. doi:10.1016/j.ejca.2005.05.009

12. Swarnakar S, Ganguly K, Kundu P, et al. Curcumin regulates expression and activity of matrix metalloproteinases 9 and 2 during prevention and healing of indomethacin-induced gastric ulcer. J Biol Chem. 2005;280:9409-9415. doi:10.1074/jbc.M413398200

13. Elburki MS, Moore DD, Terezakis NG, et al. A novel chemically modified curcumin reduces inflammation-mediated connective tissue breakdown in a rat model of diabetes: periodontal and systemic effects. J Periodontal Res. 2017;52(2):186-200. doi:10.1111/ jre. 12381

14. Moher D, Shamseer L, Clarke M, et al. Preferred reporting items for systematic review and meta-analysis protocols (PRISMA-P) 2015 statement. Syst Rev. 2015;4:1. doi:10.1186/2046-4053-4-1

15. Vandenbroucke JP, von Elm E, Altman DG, et al. Strengthening the reporting of observational studies in epi- demiology (STROBE): explanation and elaboration. Int $J$ Surg. 2004;12:1500-1524. doi:10.1016/j.ijsu.2014.07.014
16. Elburki MS, Rossa C, Guimaraes MR, et al. A novel chemically modified curcumin reduces severity of experimental periodontal disease in rats: initial observations. Mediators Inflamm. 2014;2014:959471. doi:10.1155/2014/959471

17. Curylofo-Zotti FA, Elburki MS, Oliveira PA, et al. Differential effects of natural Curcumin and chemically modified curcumin on inflammation and bone resorption in model of experimental periodontitis. Arch Oral Biol. 2018;91:42-50. doi:10.1016/j. archoralbio.2018.04.007

18. Wang HH, Lee HM, Raja V, et al. Enhanced efficacy of chemically modified curcumin in experimental periodontitis: systemic implications [published correction appears in J Exp Pharmacol. 2020 Mar 10;12:73]. J Exp Pharmacol. 2019;11:1-14. doi:10.2147/JEP. S171119

19. Deng J, Golub LM, Lee HM, et al. Chemically-modified curcumin 2.24: a novel systemic therapy for natural periodontitis in dogs. $J$ Exp Pharmacol. 2020;12:47-60. doi:10.2147/JEP.S236792

20. Elburki MS, Rossa C, Guimarães-Stabili MR, et al. A chemically modified curcumin (CMC 2.24) inhibits nuclear factor $\kappa \mathrm{B}$ activation and inflammatory bone loss in murine models of LPS-induced experimental periodontitis and diabetes-associated natural periodontitis. Inflammation. 2017;40:1436-1449. doi:10.1007/s10753-017-0587-4

21. Bebarta V, Luyten D, Heard K. Emergency medicine animal research: does use of randomization and blinding affect the results? Acad Emerg Med. 2003;10:684-687. doi:10.1111/j.1553-2712.2003. tb00056.x

22. Guimarães MR, Coimbra LS, de Aquino SG, Spolidorio LC, Kirkwood KL, Rossa C. Potent anti-inflammatory effects of systemically administered curcumin modulate periodontal disease in vivo. J Periodontal Res. 2011;46:269-279. doi:10.1111/j.16000765.2010.01342.x

\section{Publish your work in this journal}

The Journal of Experimental Pharmacology is an international, peerreviewed, open access journal publishing original research, reports, reviews and commentaries on all areas of laboratory and experimental pharmacology. The manuscript management system is completely

Submit your manuscript here: https://www.dovepress.com/journal-of-experimental-pharmacology-journal online and includes a very quick and fair peer-review system. Visit $\mathrm{http} / / / \mathrm{www}$. dovepress.com/testimonials.php to read real quotes from published authors. 